\title{
Synthesis of Molecularly Imprinting Polymers for the Removal of Xylenol Orange from Water
}

\author{
Showkat Ahmad Bhawani*†, Nur Anati Bazilah Daud*, Salma Bakhtiar*, Rachel Marcela Roland* and \\ Mohamad Nasir Mohamad Ibrahim** \\ *Faculty of Resource Science and Technology, Universiti Malaysia Sarawak (UNIMAS), Kota Samarahan, \\ Sarawak, 94300, Malaysia \\ **School of Chemical Sciences, Universiti Sains Malaysia, Pulau Pinang, 11800, Malaysia \\ †Corresponding author: Showkat Ahmad Bhawani; sabhawani@gmail.com
}

Nat. Env. \& Poll. Tech. Website: www.neptjournal.com

Received: 01-08-2019

Accepted: 07-11-2019

Key Words:

Molecularly imprinted polymers; Microemulsion; Xylenol orange

\begin{abstract}
The molecularly imprinted polymers (MIPs) were prepared by using the non-covalent approach. In the polymerization process, xylenol orange was used as a template $(\mathrm{T})$, acrylic acid as a functional monomer (M), divinylbenzene as a cross-linker ( $\mathrm{CL}$ ) and 2,2'-azobisisobutyronitrile (AIBN) as an initiator and microemulsion as a solvent. The synthesized polymers were characterized by using FTIR and SEM micrograph. The batch binding analysis was used to evaluate the rebinding efficiency of imprinted polymers. The highest rebinding efficiency was obtained from the MIP-R2 (0.1:0.6:2, T:M:CL). The selected MIP-R2 was used for the removal of xylenol orange from the water sample and have shown removal efficiency of about $80 \%$.
\end{abstract}

\section{INTRODUCTION}

The molecularly imprinting technology was first introduced by Wulff \& Sarhan (1972) and was expanded by Mosbach and coworkers in 1980s (Andersson et al. 1984). This technology enables us to synthesize the materials with highly specific receptor sites towards the target molecules. MIPs are categorized as highly cross-linked polymers and can bind target compounds with high specificity. They are synthesized in the presence of the target molecule which acts as a template (Lok \& Son 2009). MIPs have been attributed several advantages such as high selectivity and affinity, high stability and the ease of preparation (Piletsky et al. 2006). They can also be used repeatedly without loss of activity with high mechanical strength and are durable to harsh chemical media, heat and pressure as compared to biological receptors (Lavignac et al. 2004). Svenson and Nicholas (Svenson \& Nicholls 2001), have proved that polymers are thermally resilient and can retain their chemical affinity.

The most important condition to produce MIP network with high potential recognition sites is that there must be a good interaction between monomer and template. Based on the nature of pre-polymerization interactions between the template and monomer, there are two strategies employed for MIP technology. Self-assembling approach (Arshady $\&$ Mosbach 1981), similar to the biological recognition systems where non-covalent forces like hydrogen bonds, Van der Waals forces, ion or hydrophobic interaction and metal coordination were used. The most frequent approach for the preparation of MIPs is self-assembling. This is due to the simplicity of complex formation and dissociation and the flexibility where available functional monomers can interact with almost any type of templates.

MIPs have been successfully applied in various fields such as in chiral separation, solid-phase extraction, biomimetic sensor, and controlled release devices of several drugs (Caro et al. 2006). It has been used widely for the detection and treatment of water pollutants even at very low concentrations (Schreibera et al. 2009). Molecularly imprinted materials can also be used in combination with the catalyst to form novel composite adsorbent or catalyst systems.

Xylenol orange, [3,3-bis-N, N, bis-(carboxymethyl) aminomethyl-o-cresolsulfonephthalein] is used for the determination of many metal ions because it is an excellent complexometric indicator and potentiometric reagent. The effluents discharged from xylenol orange manufacturing industries and laboratories pollute water bodies. In this way, the presence of xylenol orange in the water bodies further attracts heavy metals and causes various health problems to both humans and aquatic animals. Therefore, removal of xylenol orange from the water is very important to safe- 
guard the aquatic as well as terrestrial life. The presence of dyes in water bodies interferes with the absorption of light and in other words colour is the prominent contaminant in the water. In this regard, molecular imprinting polymers are promising materials for the removal of pollutants from environmental water.

\section{MATERIALS AND METHODS}

Chemicals and reagents: The following chemicals were used in this study: Xylenol orange $\left(\mathrm{C}_{31} \mathrm{H}_{32} \mathrm{~N}_{2} \mathrm{O}_{13} \mathrm{~S}\right)$ was purchased from Acros Organics USA, 2,2'-azobis (isobutyronitrile) (AIBN) was purchased from R\&M Marketing Company (Essex, United Kingdom), cetyltrimethylammonium bromide (CTAB) from R\&M Chemicals, hexane from HmbG Chemical (Hamburg, Germany), butanol from R\&M Chemical, acrylic acid (AA) from Sigma-Aldrich, divinylbenzene (DVB) from Merck Schuchardt OHG (Hohenbrunn, Germany), acetic acid from Avantor Performance Materials Incorporated (Center Valley, Pennsylvania), acetone from HmbG Chemical and methanol from R\&M Marketing Company.

Equipment: Fourier transformed infrared (FTIR) spectrometer (Nicolet iS10), Scanning Electron Microscopy (SEM) (JEOL JSM-6390LA), Ultraviolet-Visible (UV-Vis) spectrophotometer (V-630) and (SP-830 plus), sonicator bath (2510 Branson), shaker (Multi Shaker NB-101MT), hot plate.

Preparation of microemulsion: In this study, water in oil microemulsion was used as a solvent for the synthesis of the polymer. The microemulsion was prepared by mixing 8 grams of CTAB powder, 10 gram of distilled water, 160 $\mathrm{mL}$ of hexane and $25 \mathrm{~mL}$ of butanol in a conical flask. The solution mixture was sonicated for about 20 minutes.

Preparation of MIP and NIP: The MIPs of xylenol orange were prepared by using the non-covalent approach. The composition of the two different MIPs is given in Table 1. Initially, xylenol orange was dissolved in microemulsion followed by the addition of acrylic acid, divinylbenzene and AIBN. The reaction mixture was sonicated for $15 \mathrm{~min}$ followed by the purging with nitrogen gas for $15 \mathrm{~min}$ to remove traces of oxygen. Finally, the conical flask was sealed tightly and kept in the water bath for 6 hours. Initially, the temperature was maintained at $55^{\circ} \mathrm{C}$ for the first three hours and then the temperature was increased up to $60^{\circ} \mathrm{C}$ and kept constant for the next three hours. The synthesized polymers were filtered and washed with methanol. The same procedure was repeated for the preparation of non-imprinted polymer without the template.

The template was removed from the polymer matrix by washing with methanol/acetic acid solution (10: 1, v/v, methanol and acetic acid) several times. The removal of the xylenol orange was monitored by the disappearance of xylenol orange band at $588 \mathrm{~nm}$ in UV-vis spectra.

Characterization: Scanning electron microscope (SEM) was used to observe the surface morphology of the polymer particles. Before the SEM analysis, the dried specimen was coated under vacuum with a thin layer of gold (Lian \& Wang 2012).

FTIR was used to observe the functional groups present in the polymer matrix in the range of $4000-400 \mathrm{~cm}^{-1}$ by using $\mathrm{KBr}$ pellet.

Batch binding assay: Batch adsorption experiment was performed to evaluate the binding efficiency of imprinted polymers (Komiyama et al. 2003). A 10 ppm solution was prepared from the stock solution of xylenol orange. A series of three flasks were used for three different polymers (MIP1, MIP2 and NIP). In three different flasks containing $10 \mathrm{ppm}$ of $75 \mathrm{~mL}$ solution, $50 \mathrm{mg}$ of polymers (MIP1, MIP2 and NIP) were added. After that, all the flasks were kept on the shaker and then samples were collected after different time intervals $(0,30,60,90,120,150,210$, and $270 \mathrm{~min})$. The absorbance of each extracted solution was determined by the UV spectrometry. The degree of extraction of xylenol orange was calculated using the following equation (1):

$$
\text { Extraction }(\%)=\frac{C(\text { initial })-C(\text { final })}{C(\text { initial })} \times 100
$$

Where C (initial) and C (final) are the concentrations of xylenol orange before and after extraction in the solution, respectively.

\section{Selectivity Study}

Selectivity study of MIP and NIP towards xylenol oranges dye was studied by comparing the uptake behaviour of MIP and NIP towards xylenol orange and a competitive malachite

Table 1: Composition of polymers.

\begin{tabular}{|llll|}
\hline Polymers & Xylenol Orange $(\mathrm{mmol})$ & AA $(\mathrm{mmol})$ & DVB $(\mathrm{mmol})$ \\
\hline MIP 1 & 0.1 & 0.4 & 2.0 \\
MIP 2 & 0.1 & 0.6 & 2.0 \\
NIP & 0.0 & 0.6 & 2.0 \\
\hline
\end{tabular}


green dye. The solutions of both the dyes were mixed and analysed for both MIP and NIP. The batch binding procedure was followed up to a contact time of $60 \mathrm{~min}$. The extraction efficiency of MIP and NIP were calculated by using the following equations.

$$
\mathrm{Kd}=[(\mathrm{Ci}-\mathrm{Cf}) / \mathrm{Cf} \times \mathrm{M}] \times \mathrm{V}
$$

Where,

$$
\begin{aligned}
& \mathrm{kd}=\text { the distribution coefficient } \\
& \mathrm{Ci}=\text { the initial volume of solution } \\
& \mathrm{Cf}=\text { final volume of the solutions, }
\end{aligned}
$$

Where, $\mathrm{V}$ and $\mathrm{M}$ are the volume of the solution and mass of MIP or NIP used respectively.

The selectivity coefficient (kXO-MG) was calculated by using Eq. 3 as follows:

$$
\text { ksel XO - MG }=\mathrm{KdXO} / \mathrm{Kd}, \mathrm{MG}
$$

Where, $\mathrm{KdXO}$ and $\mathrm{KdMG}$ are the distribution coefficient of xylenol orange and malachite green, respectively.

Relative selectivity coefficients, $\mathrm{K}^{\prime}$ can be calculated by using Eq. 4 .

$$
\mathrm{K}^{\prime}=\mathrm{kselMIP} / \mathrm{kselNIP}
$$

Where, kselMIP and kselNIP are the selectivity coefficients of imprinted polymers and non- imprinted polymers, respectively

\section{Removal of Xylenol Orange from Spiked Water Sample}

The water sample was collected from the river. The sample water was filtered and centrifuged to remove suspended materials. After that $40 \mathrm{~mL}$ of water was spiked with $40 \mathrm{~mL}$ of $20 \mathrm{ppm}$ of xylenol orange solution. The final concentration obtained after spiking was $10 \mathrm{ppm}$. The selected MIP was used for the removal of Xylenol orange from lake water and the same procedure was followed as adopted in the batch binding process. The absorbance of free xylenol orange was observed by the UV-vis spectrophotometry. The degree of removal of xylenol orange from the water sample was calculated by using equation 1 .

\section{RESULTS AND DISCUSSION}

The molecularly imprinted polymers for xylenol orange were prepared in microemulsion by free radical polymerization method. The microemulsion can solubilize both hydrophilic and hydrophobic compounds as compared to other organic solvents. Microemulsions are isotropic and thermodynamically stable mixture of oil, water, surfactant and usually with a co-surfactant (Shinoda \& Lindman 1987).

\section{Infrared Spectroscopy}

The IR spectra for MIPs (Ratio 1 and 2) and NIP are shown in Fig. 1, and exhibited similar characteristic peaks. These

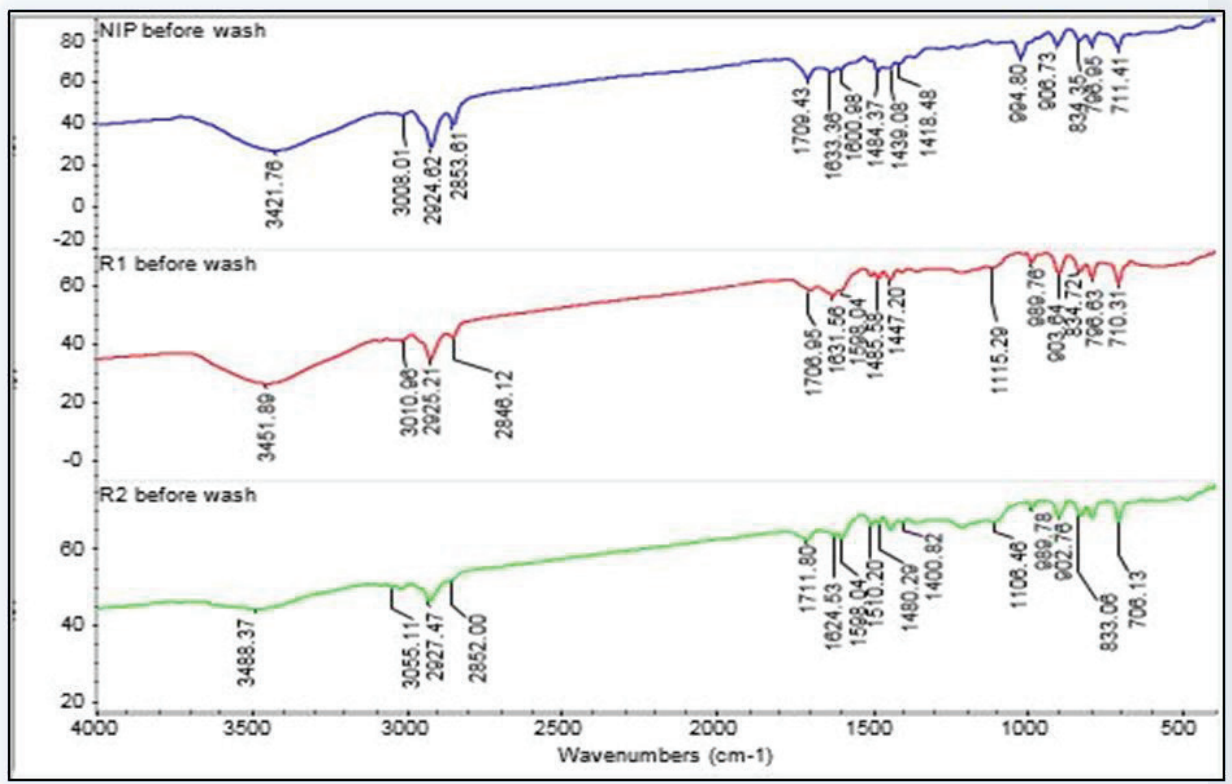

Fig. 1: FTIR spectra of MIPs (R1 and R2) and NIP. 
characteristic peaks showed a similar significant backbone structure of different polymers. These spectra show a broad stretching vibrational band allocated to hydroxyl $(\mathrm{OH})$ at about $3488 \mathrm{~cm}^{-1}-3421 \mathrm{~cm}^{-1}$ (MIP-R1, MIP-R2 and NIP), and the vibrational band of the carboxyl group $(\mathrm{C}=\mathrm{O})$ in carboxylic acid at $1711 \mathrm{~cm}^{-1}-1708 \mathrm{~cm}^{-1}$ (MIP-R1, MIP-R2 and NIP). The other absorption peaks that indicate the stretching band of vinylic $\mathrm{C}=\mathrm{C}$ bonds in the range of $1620-1680 \mathrm{~cm}^{-1}$, and C-O bonds (range of $1050-1150 \mathrm{~cm}^{-}$ ${ }^{1}$ ) as well as C-H bonds (range of 2850-2950 $\mathrm{cm}^{-1}$ ) for all the three polymers. In NIP, there is no peak in the range of $1000-1250 \mathrm{~cm}^{-1}$ which represents the amine group of the template present in MIPs. The peaks in the range of 989-902 $\mathrm{cm}^{-1}$ represent the stretching vibrations of $=\mathrm{C}-\mathrm{H}$ and $=\mathrm{CH}_{2}$ and the peak at around $833 \mathrm{~cm}^{-1}$ represents the out of plane bending of $=\mathrm{C}-\mathrm{H}$ and $=\mathrm{CH}_{2}$. If we compare all the three polymers in terms of bonding between template and monomer it is clear from the spectra that there is a shift in the wavelength from $3421 \mathrm{~cm}^{-1}$ (NIP) to $3451 \mathrm{~cm}^{-1}$ (MIP-R1) and $3488 \mathrm{~cm}^{-1}$ (MIP-R2).

\section{Morphological Study of MIP and NIP}

The morphology of the molecularly imprinted polymers was done by scanning under a scanning electron microscope (SEM). From the micrograph (Fig. 2) it is clear that the synthesized polymers are spherical and in micro size. This can be concluded that the microemulsion polymerization method can be used for the synthesis of spherical polymer particles. The regular spherical shape of polymer particles widens the applicability of this method for various environmental applications. The effectiveness of using microemulsion as a solvent has been investigated (Zhang et al. 2012) and have obtained favourable characteristic of the imprinted microspheres in better molecular recognition of MIP.

\section{Rebinding Efficiency of Polymers (MIPs and NIP)}

The binding affinity of a polymer describes the binding strength between an analyte molecule (template) and the polymer. While binding capacity describes the maximum amount of analyte that can be bound with the polymer. Both binding affinity and binding capacity are determined by batch experiment (Rachkov \& Minoura 2000). The batch binding process is used to observe the efficiency of a synthesized material in the way to determine the rebinding capacity of imprinted polymers. The rebinding results are used to evaluate the affinity and selectivity of the imprinted polymers for the template. In this study, two different ratios of monomer were used to achieve the best complementary sites for the template. The results revealed in Fig. 3 clearly show that the rebinding efficiency of MIP-R2 $(80.50 \%)$ is highest as compared to the MIP-R1 (60.50\%) and NIP (20.54\%). This observable adsorption rate was due to the preferential and rapid adsorption of template molecule onto the recognition sites in the cavities of MIPs (Deilami et al. 2010). This indicates that the MIP-R2 can be used for further study, especially in the application. This means that the MIP-R2 has more complementarily binding sites for xylenol orange. The lowest rebinding efficiency of NIP is because of the lack of binding cavities

\section{Selectivity}

Based on the results reported in Table 2, it is clear that MIP-R2 has higher selectivity towards xylenol orange template compared to malachite green. The Kd of MIP-R2 towards xylenol orange is higher than malachite green, hence, MIP-R2 was favourable to extract xylenol orange. The relative selectivity coefficient is greater than 1 that favours the selectivity of MIP-R2 towards xylenol orange.

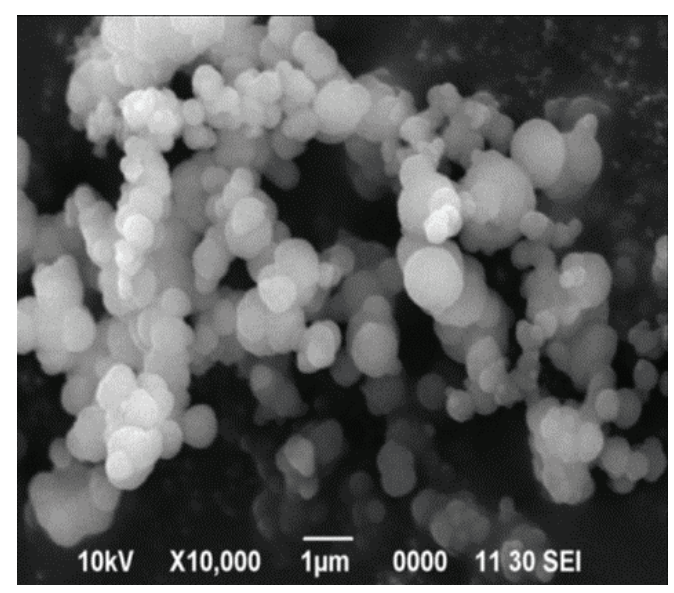

Fig. 2: SEM image of the imprinted polymer. 


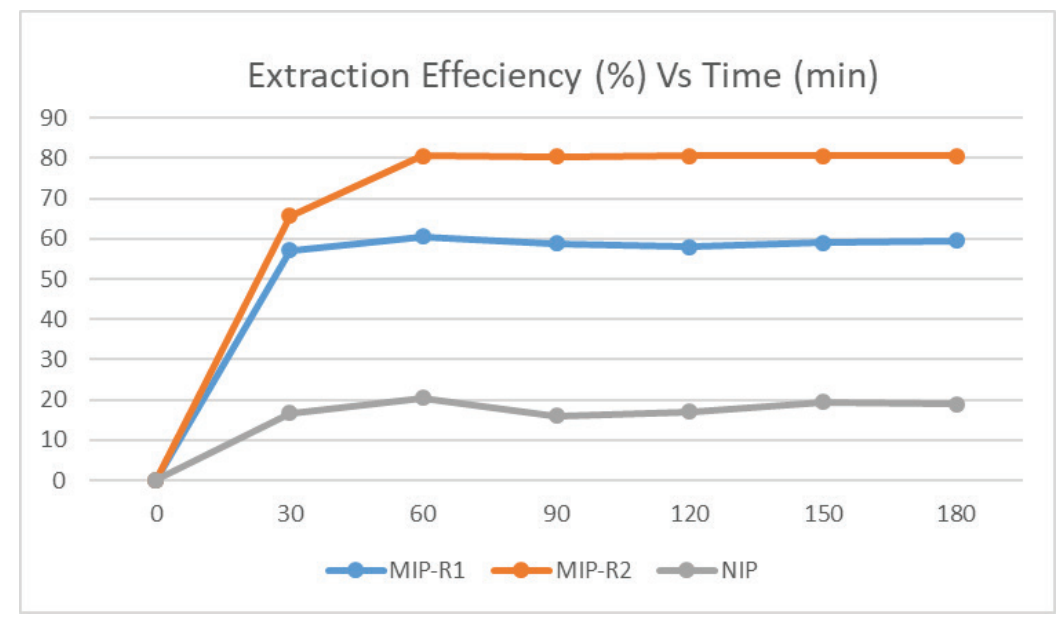

Fig. 3: Rebinding efficiency of different polymers.

Table 2: Selectivity of MIP-R2

\begin{tabular}{|lllll|}
\hline Template & KD (MIP-R2) & KD (NIP) & $k$ sel & $k$ \\
\hline Xylenol orange & 80 & 20 & 3.2 & 1.6 \\
Malachite green & 25 & 10 & 2 & \\
\hline
\end{tabular}

\section{Removal of Xylenol Orange from Spiked Water Sample}

One of the important factors is the study of the capacity of a sorbent to quantitatively remove a specific amount of dyes present in natural waters. The spiked water sample from river water was investigated for the removal of xylenol orange by using the best selected imprinted polymer. In this study, MIP-R2 and NIP were used to study the removal capacity. The results observed from the application study revealed that about $80 \%$ of xylenol orange was removed by using MIP-R2 as compared to NIP which was below $20 \%$.

\section{ACKNOWLEDGMENTS}

Authors are thankful to the Universiti Malaysia Sarawak (UNIMAS), for providing necessary research facilities.

\section{REFERENCES}

Andersson, L., Sellergren, B. and Mosbach, K. 1984. Imprinting of amino acid derivatives in macroporous polymers. Tetrahedron Letter, 25 : 5211-5214.

Arshady, R. and Mosbach, K. 1981. Synthesis of substrate-selective polymers by host-guest polymerization. Macromolecular Chemistry and Physics, 182: 687-692.

Caro, E., Marce, R.M., Borrull, F., Cormack, P.A.G. and Sherrington, D.C. 2006. Application of molecularly imprinted polymers to solid-phase extraction of compounds from environmental and biological samples. Trends Anal. Chem., 25: 143-154.

Deilami, S.A., Abdouss, M. and Seyedi, S.R. 2010. Synthesis and characterization of molecularly imprinted polymer for controlled release of tramadol. Central European Journal of Chemistry, 8(3): 687-695.

Komiyama, M., Takeuchi, T., Mukawa, T. and Asanuma, H. 2003. Molecular Imprinting from Fundamentals to Applications. Wiley-VCH, Weinheim.

Lavignac, N., Allender, C.J. and Brain, K.R. 2004. Current status of molecularly imprinted polymers as alternatives to antibodies in sorbent assays. Analytica Chimica Acta, 510: 139-145.

Lian, Z. and Wang, J. 2012. Molecularly imprinted polymer for selective extraction of malachite green from seawater and seafood coupled with high-performance liquid chromatographic determination. Marine Pollution Bulletin, 64: 2656-2662.

Lok, C. and Son, R. 2009. Application of molecularly imprinted polymers in food sample analysis - A perspective. International Food Research Journal, 16: 127-140.

Piletsky, S.A., Turner, N.W. and Laitenberger, P. 2006. Molecularly imprinted polymers in clinical diagnostics - Future potential and existing problems. Medical Engineering and Physics, 28: 971-977.

Rachkov, A. and Minoura, N. 2000. Recognition of oxytocin and oxytocin-related peptides in aqueous media using a molecularly imprinted polymer synthesized by the epitope approach. J. Chromatogr., A 889: 111.

Schreibera, T., Webera, A., Niedergalla, K., Rieglera, J., Brynioka, D., Hirtha, T. and Tovara, G.E.M. 2009. Water treatment by molecularly imprinted polymer nanoparticles. MRS Spring Meeting. Cambridge Journals Online, 11, 69.

Shinoda, K. and Lindman, B. 1987. Organised surfactant systems: Microemulsions. Langmuir, 3: 135-149.

Svenson, J. and Nicholls, I.A. 2001. On the thermal and chemical stability of molecularly imprinted polymers. Analytica Chimica Acta, 435: 19-24. 
Wulff, G. and Sharhan, A. 1972. Use of polymers with enzymes-analogous structures for the resolution of racemates. Angewandte Chemie-International Edition in English, 11: 341-344.

Zhang, H., Dramou, P., He, H., Tan, S., Pham-Huy, C., \& Pan, H. 2012.
Molecularly Imprinted Stationary Phase Prepared by Reverse Micro-Emulsion Polymerization for Selective Recognition of Gatifloxacin in Aqueous Medi. Journal of Chromatographic Science, 250, 499-508. 